Synchronous neural interactions assessed by magnetoencephalography: a functional biomarker for brain disorders

This content has been downloaded from IOPscience. Please scroll down to see the full text. 2007 J. Neural Eng. 4349

(http://iopscience.iop.org/1741-2552/4/4/001)

View the table of contents for this issue, or go to the journal homepage for more

Download details:

IP Address: 152.16.191.250

This content was downloaded on 07/07/2016 at 19:52

Please note that terms and conditions apply. 


\title{
Synchronous neural interactions assessed by magnetoencephalography: a functional biomarker for brain disorders*
}

\author{
Apostolos P Georgopoulos ${ }^{1,2,3,4,5,6,7,16}$, Elissaios Karageorgiou ${ }^{1,2}$, \\ Arthur C Leuthold ${ }^{1,2,7}$, Scott M Lewis ${ }^{1,3,7}$, Joshua K Lynch ${ }^{1,2}$, \\ Aurelio A Alonso ${ }^{1,8}$, Zaheer Aslam ${ }^{4,9}$, Adam F Carpenter ${ }^{3}$, \\ Angeliki Georgopoulos ${ }^{10,11}$, Laura S Hemmy ${ }^{4,12}$, Ioannis G Koutlas ${ }^{1,13}$, \\ Frederick J P Langheim ${ }^{1,6,7}$, J Riley McCarten ${ }^{3,12}$, Susan E McPherson ${ }^{3,9}$, \\ José V Pardo ${ }^{4,9}$, Patricia J Pardo ${ }^{1,4,7,14}$, Gareth J Parry ${ }^{3}$, \\ Susan J Rottunda ${ }^{12}$, Barbara M Segal ${ }^{10}$, Scott R Sponheim ${ }^{1,9,14}$, \\ John J Stanwyck ${ }^{15}$, Massoud Stephane ${ }^{4,9}$ and Joseph J Westermeyer ${ }^{4,9}$ \\ ${ }^{1}$ Brain Sciences Center, Veterans Affairs Medical Center, Minneapolis, MN 55417, USA \\ ${ }^{2}$ Department of Neuroscience, University of Minnesota Medical School, Minneapolis, MN 55455, USA \\ ${ }^{3}$ Department of Neurology, University of Minnesota Medical School, Minneapolis, MN 55455, USA \\ ${ }^{4}$ Department of Psychiatry, University of Minnesota Medical School, Minneapolis, MN 55455, USA \\ ${ }^{5}$ Center for Cognitive Sciences, University of Minnesota, Minneapolis, MN 55455, USA \\ ${ }^{6}$ M.D.-Ph.D. Program, University of Minnesota, Minneapolis, MN 55455, USA \\ ${ }^{7}$ Graduate Program in Neuroscience, University of Minnesota, Minneapolis, MN 55455, USA \\ ${ }^{8}$ Graduate Program in Oral Biology, University of Minnesota Medical School, Minneapolis, MN 55455, \\ USA \\ ${ }^{9}$ Mental Health Patient Service Line, Department of Veterans Affairs Medical Center, Minneapolis, \\ MN 55417, USA \\ ${ }^{10}$ Department of Medicine, University of Minnesota Medical School, Minneapolis, MN 55455, USA \\ ${ }^{11}$ Women Veterans Comprehensive Health Center, Department of Veterans Affairs Medical Center, \\ Minneapolis, MN 55417, USA \\ ${ }^{12}$ Geriatric, Research and Education Clinical Center, Department of Veterans Affairs Medical Center, \\ Minneapolis, MN 55417, USA \\ ${ }^{13}$ Department of Diagnostic and Biological Sciences, University of Minnesota School of Dentistry, \\ Minneapolis, MN 55455, USA \\ ${ }^{14}$ Department of Psychology, University of Minnesota, Minneapolis, MN 55455, USA \\ ${ }^{15}$ Research Service, Department of Veterans Affairs Medical Center, Minneapolis, MN 55417, USA \\ E-mail: omega@umn.edu (A P Georgopoulos)
}

Received 10 May 2007

Accepted for publication 4 July 2007

Published 27 August 2007

Online at stacks.iop.org/JNE/4/349

\begin{abstract}
We report on a test to assess the dynamic brain function at high temporal resolution using magnetoencephalography (MEG). The essence of the test is the measurement of the dynamic synchronous neural interactions, an essential aspect of the brain function. MEG signals were recorded from 248 axial gradiometers while 142 human subjects fixated a spot of light

\footnotetext{
* Contribution by the authors: Designed research (APG); acquired data (AAA, IGK, FJPL, ACL, SML, JJS); analyzed data (APG, EK, ACL, JKL); wrote the paper (APG, EK, ACL, SML); contributed subjects (AAA, ZA, AFC, AG, LSH, IGK, FJPL, SML, JRM, SEM, JVP, PJP, GJP, SJR, BMS, SRS, MS, JJS, JJW); discussed results (All); contributed equally (ZA, AFC, AG, LSH, FJPL, JRM, SEM, JVP, PJP, GJP, SJR, BMS, SRS, MS, JJS, JJW).

${ }^{16}$ Correspondence should be addressed to Apostolos P Georgopoulos: Brain Sciences Center (11B), Veterans Affairs Medical Center, One Veterans Drive, Minneapolis, MN 55417, USA.
} 
for 45-60 s. After fitting an autoregressive integrative moving average (ARIMA) model and taking the stationary residuals, all pairwise, zero-lag, partial cross-correlations $\left(P C C_{i j}^{0}\right)$ and their $z$-transforms $\left(z_{i j}^{0}\right)$ between $i$ and $j$ sensors were calculated, providing estimates of the strength and sign (positive, negative) of direct synchronous coupling at $1 \mathrm{~ms}$ temporal resolution. We found that subsets of $z_{i j}^{0}$ successfully classified individual subjects to their respective groups (multiple sclerosis, Alzheimer's disease, schizophrenia, Sjögren's syndrome, chronic alcoholism, facial pain, healthy controls) and gave excellent external cross-validation results.

\section{Introduction}

Like any other organ in the body, the function of the brain needs to be assessed to evaluate its status in health and disease. However, unlike any other organ in the body, no good tests of brain function are available. Typically, one relies on behavioral examinations, including a neurological examination, psychiatric interview, and neuropsychological testing. In addition, several methods exist to assess the brain structure (e.g. magnetic resonance imaging, MRI), chemistry (e.g. magnetic resonance spectroscopy, fluorodeoxy-glucose-based positron emission tomography, PET), pharmacology (e.g. ligand-based PET), and brain activation patterns based on long (e.g. seconds or minutes) acquisition times (e.g. functional MRI and $\mathrm{O}^{15}$ based PET). With respect to biomarkers for brain disease, most of the ongoing effort has focused on biological substances (proteins, genes, etc). Although potentially useful, this approach cannot provide direct information concerning the functional status of the brain. This can be assessed only by methods suitable for recording dynamic brain activity with a high (e.g. millisecond) temporal resolution, such as electroencephalography [1-3] and magnetoencephalography (MEG) [4, 5].

Ideally, a test for assessing brain function should be noninvasive, passive, short, simple, dynamic, robust and sensitive to changes in brain function. It should also evaluate interactions among neuronal populations, since this is the essence of brain function. In a recent study [6] we reported on synchronous neural interactions revealed by MEG while subjects fixated a spot for $45 \mathrm{~s}$. Based on the fact that these interactions were very similar across subjects, we proposed that this robustness points to a relatively stable synchronicity pattern among neural populations which could, in turn, serve as a canonical network for assessing dynamic brain function. In the present study, we tested this idea by using these interactions as classifiers of brain status and assessing their potential as biomarkers for several brain diseases in a total of 142 subjects. For that purpose, we initially studied 52 subjects to derive discriminant classification functions. These functions were subsequently applied to a new group of 46 subjects in an external cross-validation procedure. Finally, 44 additional subjects were most recently incorporated for a complete sample of 142 subjects. We found that synchronous neural interactions successfully classified brain status and gave excellent external cross-validation results.

\section{Materials and methods}

\subsection{Subjects}

A total of 142 human subjects participated in this study as paid volunteers. The study protocol was approved by the relevant institutional review boards and informed consent was obtained prior to the study. There were seven groups, including healthy controls (HC), patients with Alzheimer's disease (AD), schizophrenia (SZ), chronic alcoholism (CA), Sjögren's syndrome (SS), multiple sclerosis (MS) and facial pain (FP). The composition of each group was as follows. HC ( $N=89$ (48 men, 41 women), age (mean \pm SEM) $43.7 \pm 1.7$, range: $10-82$ years); $\mathrm{AD}(N=9$ men, age $74.0 \pm 2.1$ years, average mini-mental state examination (MMSE) score $21.13 \pm 1.5) ; \mathrm{SZ}$ ( $N=16$ (13 men, 3 women), age $45.8 \pm$ 2.5 years); CA ( $N=3$ men, age $57.3 \pm 0.9$ years); SS ( $N=10$ ( 1 man, 9 women $)$ age $54.8 \pm 3.2$ years); MS $(N=12$ ( 4 men, 8 women), age $40.7 \pm 3.3$ years, secondary progressive or relapsing remitting forms); $\mathrm{FP}(N=3$ women, age $47.3 \pm 6.5$ years, arthromyalgia). Subjects belonging to a patient group had a functional brain disorder, and their diagnoses were made by a specialist in the respective field of medicine, as follows. $\mathrm{AD}$ patients were diagnosed on the basis of an interdisciplinary consensus diagnosis conference and determined to meet the criteria for (i) a diagnosis of dementia according to DSM-IV [7] and (ii) possible or probable AD according to NINCDS-ARDA criteria [8]. SZ patients were diagnosed on the basis of DSM-IV criteria [7], had no history of electroconvulsive therapy, no head trauma (overnight hospitalization or unconscious for $>5 \mathrm{~min}$ ), no past substance dependence, no current substance/alcohol dependence or abuse, and no medical conditions that effect the central nervous system (e.g. epilepsy). CA patients had not taken alcohol for $24 \mathrm{~h}$ preceding the study and had tested alcohol-free using a breath analyzer. SS patients were diagnosed on the basis of the classification criteria by the American-European consensus group for Sjögren's syndrome [9]. They complained of cognitive dysfunction, verified clinically by their physicians and by neuropsychological measurements. MS patients met the modified McDonald criteria [10], had greater than or equal to $10 \mathrm{~T} 2$ cerebral lesions, were at least 30 days post-relapse or steroid burst, and had a clear MS subtype. FP patients were diagnosed with temporomandibular joint arthralgia and myofascial pain of the masticatory muscle (arthromyalgia). Finally, the control group comprised age-matched subjects to the patient groups, as well as additional healthy subjects. 
All subjects, except for those belonging to the control group, received medications relevant to their brain illness; some of these medications were psychotropic.

For purposes of external cross-validation, we analyzed two consecutive sub-samples of subjects based on an arbitrary time point unrelated to data analysis. The first sample comprised 52 subjects (6 groups) and consisted of the following groups: $\mathrm{HC}$ ( $N=25$ (17 men, 8 women), age $47.0 \pm 3.6$, range: $23-82$ years); $\mathrm{AD}(N=6$ men, age $76.8 \pm$ 1.8 years); SZ ( $N=10$ (7 men, 3 women), age $48.2 \pm$ 2.9 years $) ; \mathrm{CA}(N=3$ men, age $57.3 \pm 0.9$ years $) ; \mathrm{SS}$ ( $N=4$ women, age $56.3 \pm 5.2$ years $)$, MS $(N=4$ ( 2 men, 2 women), age $42.5 \pm 6.4$ years). The second sample comprised 46 subjects (5 groups, the same as above except CA) whose data were processed following the first sample. This sample consisted of the following groups: $\mathrm{HC}(N=33$ (15 men and 18 women), age $36.8 \pm 2.8$, range: $11-67$ years); $\operatorname{AD}(N=2$ men, age $76.0 \pm 3.0$ years $(73,79)) ; \mathrm{SZ}(N=$ 2 men, age $30.0 \pm 2.0$ years $(27,33))$; SS $(N=5$ ( 1 man, 4 women), age $51.4 \pm 4.5$ years $)$; $M S$ ( $N=4$ (2 men, 2 women), age $36.8 \pm 5.2$ years).

\subsection{Task-data acquisition}

Our goal was to have the brain in a stable condition without engaging in any specific task. For that purpose, subjects lay supine in the MEG instrument and fixated their eyes on a spot, $\sim 62 \mathrm{~cm}$ in front of them, for 45-60 s (in different subjects) while MEG data were acquired from 248 axial gradiometers (sampled at $1017 \mathrm{~Hz}$, filtered 0.1-400 Hz; Magnes 3600WH, 4D Neuroimaging, San Diego, CA). This yielded, for each subject, a data set consisting of 248 time series with 45000 60000 time points. The cardiac artifact was removed from each series using event-synchronous subtraction [27].

\subsection{General data analysis (figure 1)}

All analyses described below were performed on singletrial, unsmoothed and unaveraged data. To compute the zero-lag cross-correlation between MEG sensor time series, individual series were made stationary by 'prewhitening' [11], for nonstationarities in the series can lead to erroneous associations [11-13]. Therefore, the first step in our analyses was to model the time series and derive stationary (or quasi-stationary) residuals from which to compute pairwise association measures, such as cross-correlations [14]. Previous work $[6,14]$ showed that an autoregressive integrative moving average (ARIMA) model of 25 AR orders, firstorder differencing, and first-order MA was adequate to yield residuals that were practically stationary with respect to the mean, variance, and autocorrelation structure. Residuals were estimated using the SPSS statistical package (SPSS for Windows, version 15, SPSS Inc., Chicago, IL, 2006). The zero-lag cross-correlation between pairs of stationary residuals was computed using the DCCF routine of the IMSL statistical library (Compaq Visual Fortran Professional edition version 6.6B). From these, the partial zero-lag crosscorrelation $P C C_{i j}^{0}$ between the $i$ and $j$ sensors was computed for all sensor pairs. $P C C_{i j}^{0}$ was transformed to $z_{i j}^{0}$ using

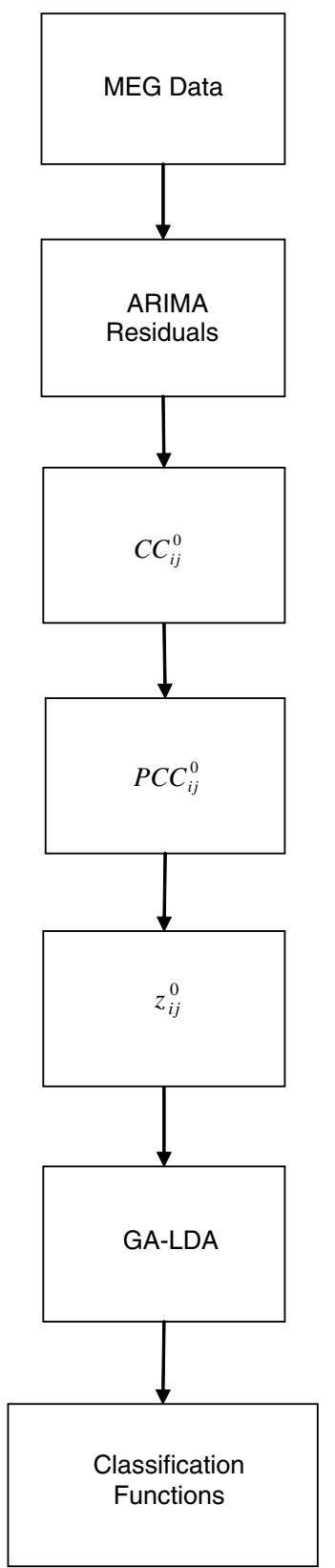

Figure 1. MEG data processing flowchart. Raw MEG data are initially cardiocorrected and subjected to an ARIMA modeling to obtain practically stationary residuals. From these, synchronous cross-correlations and partial cross-correlations are then calculated and fed into the genetic algorithm-linear discriminant analysis (GA-LDA) after $z$-transformation.

Fisher's $z$-transformation [15] to normalize its distribution:

$$
z_{i j}^{0}=0.5\left[\ln \left(1+P C C_{i j}^{0}\right)-\ln \left(1-P C C_{i j}^{0}\right)\right] .
$$

Univariate analyses of covariance (ANCOVAs) were performed on the data of each individual sensor pair for each sample, where $z_{i j}^{0}$ was the dependent variable and the gender (binary variable) and age were covariates. To assess the congruence of the distribution of the group effect among sensor pairs in the first and second sample, we coded the presence or absence of a significant effect for a given sensor pair as 1 and 0 , respectively, and computed the $\chi^{2}$ test statistic. 


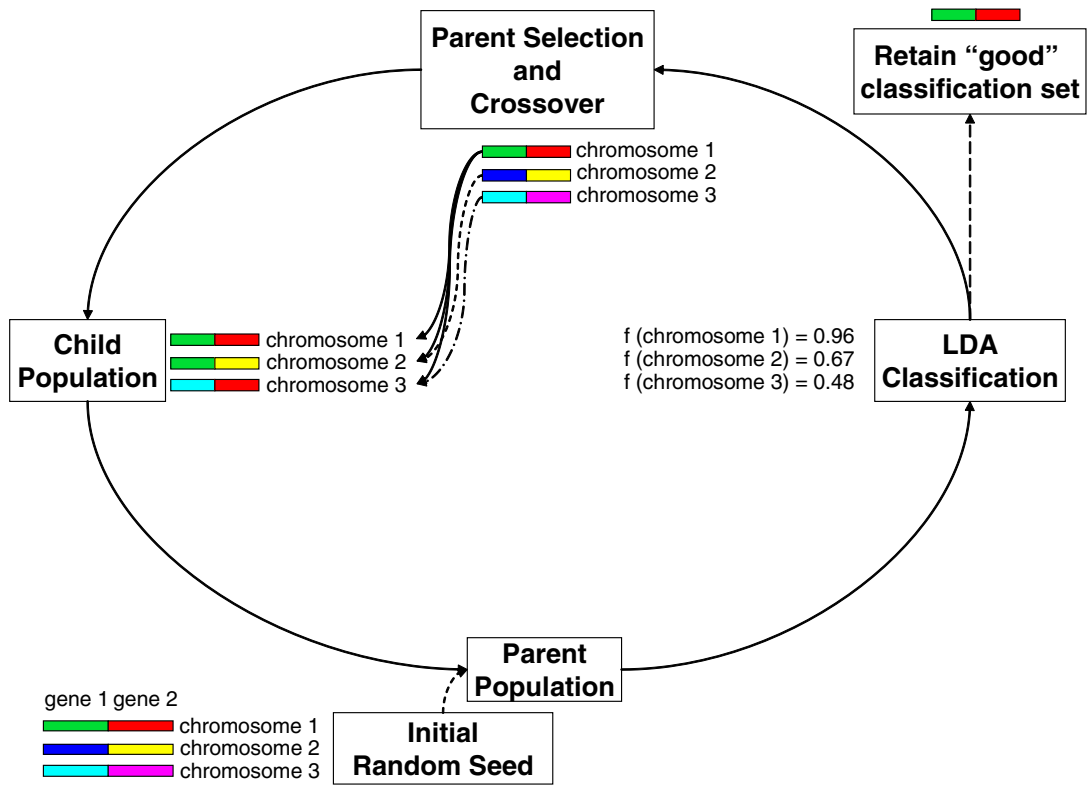

Figure 2. Genetic algorithm-linear discriminant analysis. An initial seed is used to create the first parent generation of chromosomes (possible sets) of genes $\left(z_{i j}^{0}\right)$. Chromosomes yielding good classifications are retained based on the classification results from the LDA (posterior probabilities). Each chromosome in the child generation is born from two parent chromosomes, which are probabilistically selected on the basis of their classification scores. Additionally, the best chromosome from each generation is carried over to the child generation (elitism). Finally, the child generation becomes the next parent generation.

\subsection{Linear discriminant classification analysis}

This analysis [16] was used to determine whether subsets of $z_{i j}^{0}$ exist that would correctly classify subjects into their respective groups. In this analysis, Fisher's group discrimination and subject classification were done in a canonical discriminant function (CDF) space. CDF is a weighted linear sum of the predictor variables and is derived by the statistical analysis. For $L$ groups (assuming $L$ is smaller than the number $k$ of predictors in a subset), there are $j=L-1$ discriminant functions, $\mathrm{CDF}_{i}$, where $i=1,2, \ldots, j$. Group discrimination (and individual subject classification) is performed within this multidimensional CDF space. This analysis yields group classification functions and posterior probabilities of classification of each subject to a particular group. In addition, we used a forward stepwise linear discriminant analysis on the total sample of 142 subjects (program 7M of the BMDP Dynamic, version 7, statistical package, Los Angeles, CA, 1992) to derive a single subset of predictors. The default $F$-values of the program were used $(F$-to-add-a-predictor $=$ 4.0, $F$-to-remove-a-predictor $=3.996)$. The input predictors for that analysis were $z_{i j}^{0}$ values from 271 sensor pairs which showed a highly significant group effect in an ANOVA $(P<0.001, F$-test $)$. This was done in an effort to reduce the large predictor space consisting of 30628 values.

\subsection{Genetic algorithm (GA)}

A major objective of this work was to identify successful predictor subsets from a very large space. A bruteforce approach in identifying all such subsets would be computationally deterring even for more than a few predictors, given the large size of the predictor set $(N=30628)$. For

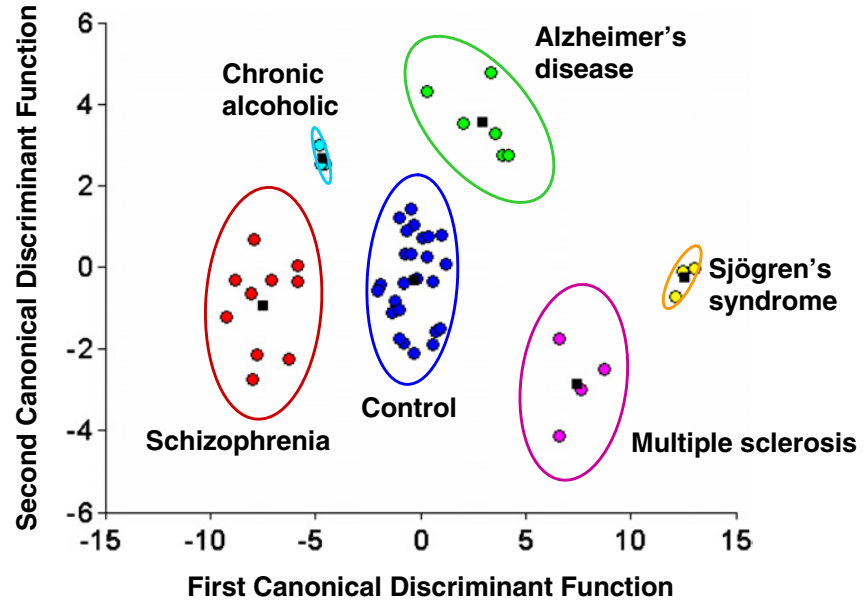

Figure 3. Classification results from 52 subjects (first sample) belonging to six groups are plotted in the two-dimensional space of the first two (out of five) canonical discriminant functions (CDF) of $20 z_{i j}^{0}$ predictors. Ellipses represent $95 \%$ confidence intervals on the center of the group centroids. The classification was $100 \%$ correct. It can be seen that all groups were clearly distinguished with no overlap in the CDF space of the first two CDFs. The null hypothesis of equality of the seven group centroids in the original, raw-data 20-dimensional $z_{i j}^{0}$ predictor space was tested using a multivariate analysis of variance and was rejected at a high level of significance $\left(P<10^{-31}\right.$, Hotteling's trace test $)$.

this reason, we used a GA [17-19] (figure 2) to reduce the computational time and optimize our search as follows. An initial subset of a certain number of $z_{i j}^{0}$ predictors (out of the 30628 available) was chosen randomly, and a micro-GA of population size $=5$ and uniform crossover was let to run for $24 \mathrm{~h}$. If, during the search, the fitness function solution 
was constant for $2 \times 10^{5}$ generations, a new random subset would be chosen and the operation repeated. Practically, a GA abides to the natural selection law of 'survival of the fittest'. This way, characteristics that improve an evaluation function (classification in this case) are maintained, whereas the ones that do not are discarded.

\subsection{Statistical analyses of the first sample}

These analyses had two objectives, namely (i) to test the hypothesis that the number of good predictor subsets exceeds that expected by chance, and (ii) to generate classification functions for the purpose of cross-validation with the second sample.

With respect to chance outcomes, given $M$ subjects belonging to $L$ groups, and subsets of the same size of $k$ predictors out of $N(=30628)$ possible, the expected number $s$ of subsets that would give $100 \%$ correct classification of each subject to its group by chance is

$$
s=Q\left(\frac{1}{L}\right)^{M}, \quad Q=\frac{N !}{k !(N-k) !} .
$$

For the analysis using an exhaustive search, we used $k=2, L=3, M=17$ (6 patients with $\mathrm{AD}, 3$ subjects with $\mathrm{CA}$, and 8 matched $\mathrm{HC}$ ) to perform a linear discriminant analysis using the robust leave-one-out method. A $100 \%$ correct classification of every subject to its corresponding group was required to retain a specific subset.

\subsection{Linear discriminant classification analysis: external cross-validation}

The linear discriminant analysis yields classification functions for each group which are then used to classify individual subjects to a group. For the external cross-validation, we used classification functions derived from the first sample to classify subjects from the second sample. For that purpose we used classification functions derived from sets of $13-16 z_{i j}^{0}$ predictors that gave $100 \%$ correct classification for the first sample and applied them to the second sample.

\section{Results}

\subsection{The first sample (52 subjects, 6 groups): initial analyses}

First, a statistically significant group effect on $z_{i j}^{0}(P<0.05$, $F$-test, ANCOVA) was found in $18 \%$ of sensor pairs. Next, we carried out a linear discriminant classification analysis (using GA) on subsets of $z_{i j}^{0}$ to find out whether individual subjects can be successfully classified to their respective groups. Indeed, we found many (in the thousands) such subsets of $z_{i j}^{0}$ predictors that classified each of the 52 subjects $100 \%$ correctly. (The exact number of all such subsets is practically impossible to determine.) An example is shown in figure 3. In many cases, successful subsets yielded not only $100 \%$ correct classification but also high (e.g., >0.98) posterior probabilities of correct classification of each subject to its group.

Since many $z_{i j}^{0}$ subsets are possible, it is useful to know whether the number of successful subsets exceeds that expected by chance. This number is typically very large and depends on the number of actual predictors, $k$, in a subset, the ensemble size of all possible $z_{i j}^{0}$ predictors $(N=30628)$, the number of groups, and the number of subjects to be classified. We investigated this problem in two tractable ways. In the first analysis, we performed an exhaustive search on a smaller sub-sample of the data using just two predictors (see section 2.6). This exhaustive assessment yielded 560 subsets with $100 \%$ correct classification (using the robust leave-oneout method), as compared to $\sim 4$ sets (3.63 to be exact) expected by chance. These proportions differ significantly (binomial theorem, normal deviate $z=23.4, P<10^{-50}$ ), indicating an excess (above chance) of good sets. In the second analysis, we used the whole first sample of 52 subjects using ten predictors, for which an exhaustive search would be infeasible. The expected number of subsets yielding 100\% correct classification by chance is essentially zero ( 0.0069 to be exact). However, our discriminant classification program yielded 79 sets with $100 \%$ correct classification after running for a while. Although we cannot calculate the exact proportion of good sets in our data (since an exhaustive search is not feasible), this proportion already exceeds appreciably the chance expectation $\left(z=8.78, P<10^{-50}\right)$.

\subsection{The second consecutive sample (46 subjects, 5 groups): external cross-validation}

In order to evaluate the robustness of this analysis and its potential as a useful clinical test, we analyzed data from 46 subjects which were processed during a subsequent period of time, following the first sample. Specifically, we wanted to find out whether the results of the analysis of the second sample were congruent with those of the first sample with respect to (a) the distribution of the group effect assessed by ANCOVA on individual $z_{i j}^{0}$ of sensor pairs $(N=30628)$, and (b) the classification outcomes of the second sample based on classification functions derived from the first sample (external cross-validation). With respect to the former, a statistically significant group effect on $z_{i j}^{0}(P<0.05, F$-test, ANCOVA) was found in $11 \%$ of sensor pairs, as compared to $18 \%$ in the first sample; the distribution of this effect among sensor pairs was highly congruent in the two samples $\left(P<10^{-11}, \chi^{2}\right.$ test). And with respect to the latter, many of the $z_{i j}^{0}$ subsets which had given $100 \%$ classification in the first sample also gave excellent classification ( $>90 \%$ correct in the thousands, $>95 \%$ correct in the hundreds) when all classification functions calculated from the first sample were applied to the second sample. These results underscore the similarity of the two samples and document the occurrence of excellent external cross-validation results.

\subsection{The total current sample (142 subjects, 7 groups)}

Recently, data from 44 additional subjects were analyzed for a total current sample of 142 subjects (7 groups). We readily identified many $z_{i j}^{0}$ predictor subsets yielding $100 \%$ correct classification of every subject to its respective group. The number of such subsets was in the thousands (for 20 
predictors), and even as few as $16 z_{i j}^{0}$ predictors would give $100 \%$ correct classification results. It is also noteworthy that the posterior probabilities of subject classification were $>0.95$ in most cases, underscoring the power of this approach. This analysis is ongoing. Finally, a stepwise discriminant analysis (see section 2.4) yielded a subset of $12 z_{i j}^{0}$ predictors which correctly classified $86.6 \%$ of the 142 subjects to their respective groups. Two cross-validation results were also obtained with this set. First, a jackknifed classification obtained by the leave-one-out method gave $78.9 \%$ correct classification. Second, the program was run ten times using $80 \%$ of the data (randomly selected) for calculating the classification functions by which to predict the group assignment of the remaining $20 \%$ of the subjects. The average correct classification was $86.4 \%$ (range: $79.3-93.8 \%$ ), and the average correct jackknifed classification was $77 \%$ (range: $72.1-83.6 \%$ ). These findings show that a high percentage of correct classification can be obtained robustly.

\section{Discussion}

Early pioneering work using quantitative EEG went a long way in laying the foundation for the application of discrimination and classification of electrophysiological brain patterns in health and disease [1-3]. Conceptually, our approach follows those early leads but factually it differs substantially, for (i) it utilizes much more accurate technology (MEG versus EEG), (ii) it is based on single (versus averaged) trials, (iii) its basic tool is a relational measure between sensor signals (crosscorrelation) instead of raw (e.g. signal amplitude) or derived (e.g. spectral power in particular frequency band) measures within individual sensors, and (iv) this cross-correlation is computed from stationary time series (after prewhitening of the raw neural signal) so that it reflects true moment-to-moment neural interactions. In addition, (v) we measure the strength of synchronicity at high temporal resolution $(1 \mathrm{~ms})$ and (vi) partial out interactions of a given pair with the rest of the neural network, so that the resulting partial zero-lag crosscorrelations are not contaminated by collinearities.

The measure underlying the successful classification obtained in this study is the pairwise zero-lag partial correlation of the MEG time series. The set of all such correlations between the 248 MEG sensors characterizes dynamic synchronicities in a large neural network. The present results suggest that these synchronicities might be regulated, since their alteration even within small subsets is a powerful discriminant of brain disease states. This idea is in accord with our previous observation that the brain pattern of partial correlations is very similar among healthy subjects [6]. It is also in keeping with the broader concept of synchronicity as a basic functional substrate for cortical function [20] and its disorders [21], as well as for higher brain function [22, 23]. The mechanisms for weak local cortical synchronization may rely on recurrent collaterals of pyramidal tract cells [24] and specific parvalbumin-immunoreactive thalamocortical neurons [25], whereas calbindin-immunoreactive thalamocortical neurons could be responsible for larger-scale, multifocal cortical synchronization [25]. The present findings suggest that finegrain synchronicity may be a fundamental aspect of cortical function that can be differentially disrupted by different disease processes, yielding a disease-specific signature.

A different issue concerns the specific subsets of zero-lag partial cross-correlations that yield high classification rates. These cannot be found by an exhaustive search, given the large space of 30628 values available and the combinatorial nature of the subsets problem. Instead, we have adopted a few different approaches in an effort to identify and evaluate such 'good' subsets. First, we used classical statistics in applying a stepwise linear discriminant analysis to identify a single subset of predictors. Since the whole set is very large, we reduced it by first carrying out an analysis of variance with the disease ('group') as a fixed factor, and then performing the stepwise linear discriminant analysis on 271 (out of 30628 ) predictors which showed a highly significant group effect. This analysis yielded a set consisting of 12 predictors with high classification rates in standard reclassification analysis as well as in jackknifed leave-oneout classification and in an $80 / 20 \%$ random split. However, like any stepwise procedure, the stepwise linear discriminant analysis relies on specific criteria for entering and/or removing predictors from the equation at each step, and these criteria can have a major influence on the outcome, in addition to the direction of stepping (forward or backward). This analysis, although useful, may not be optimally suited to our specific application. For that purpose, we first tried to identify the ideal ( $100 \%$ classification) predictor subsets by brute force, i.e. by searching within the entire combinatorial predictor subset space. We placed emphasis on small subset sizes $(<20)$ to avoid overfitting. Our initial analyses using random search did not produce any interesting result after running the program for several days. Therefore, we then implemented a genetic search algorithm to quickly locate ideal sets. Indeed, this algorithm yielded a good number of such subsets within a day. This number exceeded that expected by chance, as we found out (a) in an exhaustive search of data from a few diseases and few predictors, and (b) in a larger sample where we found that the number of ideal predictor subsets exceeded that of chance, although the exact number of such subsets could not be determined. The next step was to evaluate the power of such ideal predictor subsets to classify new subjects in an external cross-validation scheme. By focusing the genetic algorithm on that problem, we identified thousands of subsets yielding excellent cross-validation rates ( $>90 \%$; hundreds $>95 \%$ ). Due to the large search space, the exact number of such subsets cannot be known.

In summary, these results demonstrate that (a) adequate information exists in the zero-lag partial correlations to differentiate brain disease states, (b) this information can be successfully extracted using linear discriminant analysis, (c) the results exceed those expected by chance alone, and (d) the results are robust and, to a good extent, cross-validated. The long-term utility of such subsets in classifying new subjects remains to be assessed in a phase II study according to the Early Detection Research Network biomarker development protocol, within the context of a large population-based casecontrol study [26]. It should be made clear that such studies, 
at whatever stage, are always evolving, since the addition of new study subjects and new disease groups will inevitably necessitate an updating of the predictor subsets and associated classification functions. In addition, other classification methods (e.g. based on the support vector machine) could be tried and/or developed to improve the classification outcome. Finally, it should be mentioned that, although the analyses described above were applied on seven groups, they can also be applied, in general, on any pair of groups to serve, for example, as a screening test (healthy controls versus all patients) or as a more specific aid in differential diagnosis between particular brain disorders (e.g. MS versus MS-mimetic disorders, etc).

\section{Acknowledgments}

This work was supported by the Department of Veterans Affairs, the American Legion Brain Sciences Chair, and the following additional sources to individual investigators. A P Georgopoulos: Translational Grant from the Academic Health Center of the University of Minnesota. The following grants provided broad support for recruiting patients and matched control subjects: Z Aslam: Minnesota Medical Foundation. I G Koutlas: Grant-in-aid, University of Minnesota Graduate School. S Sponheim: Department of Veterans Affairs Clinical Science Research Division (Merit Review), Minnesota Medical Foundation, and the National Institute of Mental Health (5R24MH069675-03). M Stephane: Advanced Research Career Development Award, US Department of Veterans Affairs. J V Pardo: NIH grant RO1AG120852. A P Georgopoulos has a financial interest in the technology described in this publication.

\section{References}

[1] John E R et al 1977 Science 196 1393-410

[2] John E R, Prichep L S, Fridman J and Easton P 1988 Science 239 162-9

[3] John E R and Prichep L S 2006 Clin. EEG Neurosci. 37 135-43
[4] Bosboom J L, Stoffers D, Stam C J, van Dijk B W, Verbunt J, Berendse H W and Wolters E Ch 2006 Clin. Neurophysiol. 117 2521-31

[5] Stam C J, Jones B F, Manshanden I, van Cappellen van Walsum A M, Montez T, Verbunt J P, de Munck J C, van Dijk B W, Berendse H W and Scheltens P 2006 Neuroimage 32 1335-44

[6] Langheim F J P, Leuthold A C and Georgopoulos A P 2006 Proc. Natl Acad. Sci. USA 103 455-9

[7] American Psychiatric Association 2000 Diagnostic and Statistical Manual of Mental Disorders 4th edn (Washington, DC: APA)

[8] McKhann G, Drachman D, Folstein M, Katzman R, Price D and Stadlan E M 1984 Neurology 34 939-44

[9] Vitali C et al 2002 Ann. Rheum. Dis. 61 544-8

[10] Polman C H et al 2005 Ann. Neurol. 58 840-6

[11] Box G E P and Jenkins G M 1970 Time Series Analysis: Forecasting and Control (San Francisco, CA: Holden Day)

[12] Jenkins G M and Watts D G 1968 Spectral Analysis and Its Applications (San Francisco, CA: Holden Day)

[13] Priestley M B 1981 Spectral Analysis and Time Series (London, UK: Academic)

[14] Leuthold A C, Langheim F J P, Lewis S M and Georgopoulos A P 2005 Exp. Brain Res. 164 211-22

[15] Snedecor G W and Cochran W G 1989 Statistical Methods (Ames, IA: Iowa State Univ. Press)

[16] Green P E 1978 Analyzing Multivariate Data (Hinsdale, IL: Dryden Press)

[17] Mitchell M 1999 An Introduction to Genetic Algorithms (Cambridge, MA: MIT Press)

[18] Goldberg D E 1989 Genetic Algorithms in Search, Optimization and Machine Learning (Boston, MA: Addison-Wesley)

[19] Carroll D L FORTRAN Genetic Algorithm (GA) Driver (http://cuaerospace.com/carroll/ga.html)

[20] Bruno R M and Sakmann B 2006 Science 312 1622-7

[21] Uhlhaas P J and Singer W 2006 Neuron 52 155-68

[22] Singer W 1999 Neuron 24 49-65

[23] von der Malsburg C 1999 Neuron 24 95-104

[24] Stefanis C and Jasper H 1964 J. Neurophysiol. 27 855-77

[25] Jones E G 2001 Trends Neurosci. 24 595-601

[26] Pepe M S 2005 Stat. Med. 24 3687-96

[27] Leuthold A C 2003 Removal of cardiac artifact from MEG data: template subtraction revisited 33rd Ann. Meeting of the Society for Neuroscience (New Orleans, LA, 8-12 Nov. 2003) 